\title{
Gene Targeting of Desrt, a Novel ARID Class DNA-Binding Protein, Causes Growth Retardation and Abnormal Development of Reproductive Organs
}

\author{
Mireille H. Lahoud, ${ }^{1}$ Sika Ristevski, ${ }^{1}$ Deon J. Venter, ${ }^{3,4}$ Lars S. Jermiin, 5,8 \\ Ivan Bertoncello, ${ }^{3}$ Silva Zavarsek, ${ }^{1}$ Sue Hasthorpe, ${ }^{6}$ John Drago, ${ }^{7}$ David de Kretser, ${ }^{2}$ \\ Paul J. Hertzog, ${ }^{1,10,11}$ and Ismail Kola ${ }^{1,9}$ \\ ${ }^{1}$ Centre for Functional Genomics and Human Disease, and ${ }^{2}$ Molecular Reproduction and Endocrinology, Monash Institute of \\ Reproduction and Development, Monash University, Melbourne, Victoria, Australia; ${ }^{3}$ Peter MacCallum Cancer Institute, East \\ Melbourne, VIC 3002, Australia; ${ }^{4}$ Department of Pathology, University of Melbourne, Parkville, VIC 3052, Australia; ${ }^{5}$ John \\ Curtin School of Medical Research, Australian National University, Canberra, ACT 0200, Australia; ${ }^{6}$ F. Douglas Stephens \\ Surgical Research Laboratory, Royal Children's Hospital, Parkville, VIC 3052, Australia; ' Department of Medicine, Monash \\ University, Monash Medical Centre, Clayton, VIC 3168, Australia
}

\begin{abstract}
We have cloned and characterized a novel murine DNA-binding protein Desrt, with a motif characteristic of the ARID (A-T rich interaction domain) family of transcription factors. The Desrt gene encodes an 83-kD protein that is shown to bind DNA and is widely expressed in adult tissues. To examine the in vivo function of Desrt, we have generated mice with a targeted mutation in the ARID domain of Desrt. Homozygous mutants have reduced viability, pronounced growth retardation, and a high incidence of abnormalities of the female and male reproductive organs including cryptorchidism. This may thus serve as a model to dissect the mechanisms involved in the development of the reproductive tract including testicular descent. Gene-targeted mice also display a reduction in the thickness of the zona reticularis of the adrenal gland and transient aberrations of the $\mathrm{T}$ and $\mathrm{B}$ cell compartments of primary lymphoid organs. These data show that this novel DNA-binding protein, Desrt, has a nonredundant function during growth and in the development of the reproductive system.
\end{abstract}

To identify novel genes involved in early stages of mammalian development, we generated and characterized genes expressed in a murine blastocyst cDNA library (Corrick et al. 1996). One of the clones (BL21) identified from this library was highly expressed in the blastocyst and appeared to be developmentally regulated as it was induced by retinoic acid treatment of an embryonal carcinoma cell line. Furthermore, part of the BL21 sequence had high similarity with part of the AT-rich interaction domain (ARID).

The ARID domain defines a highly conserved family of sequence-specific DNA-binding proteins (Herrscher et al. 1995; Gregory et al. 1996) that appear to play a role in diverse biological functions including cell proliferation, differentia-

Present addresses: ${ }^{8}$ Australian Genomic Information Centre and School of Biological Sciences, The University of Sydney, Sydney, NSW 2006, Australia; 'Pharmacia Corporation, Kalamazoo, Michigan 49007, USA.

${ }^{10}$ Monash Institute of Reproduction and Development, Monash Medical Centre, 246 Clayton Road, Clayton, Victoria 3168, Australia.

1 Corresponding author.

E-MAIL paul.hertzog@med.monash.edu.au; FAX 61-3-95947211. Article published on-line before print: Genome Res., 10.1101/gr. 168801. Article and publication are at http://www.genome.org/cgi/doi/10.1101/ gr.168801. tion, and development. The ARID domain was initially identified in the mouse Bright ( $\underline{B}$ cell regulator of IgH transcription) protein and subsequently in yeast Swi1, Drosophila dri, osa/eld, mouse Jumonji, and human Jumonji, MRF2, DRIL1, RBP1, RBP2, p270 and Xe169, and SMCY. Bright and dri have both been shown to bind DNA and regulate transcription (Herrscher et al. 1995; Gregory et al. 1996; Valentine et al. 1998). Swi1 and p270 are components of the Swi/Snf complex (Peterson et al. 1994; Dallas et al. 1998), which is also implicated in transcriptional regulation (Chiba et al. 1994). Drosophila dri, eld/osa, and mouse Jumonji are required for embryonic development and survival (Takeuchi et al. 1995; Treisman et al. 1997; Shandala et al. 1999; Vazquez et al. 1999).

In this study we describe the isolation and sequencing of a novel ARID family member that is widely expressed and encodes a DNA-binding protein. Mutant mice generated by gene targeting have reduced viability, are severely growth retarded, display transient immune aberrations, have a reduced zona reticularis of the adrenal gland, and display abnormalities in the development of the reproductive organs including cryptorchidism. Hence, this novel gene has been named Desrt on the basis of the observed phenotype of the mutant mice (developmentally and sexually retarded with transient immune abnormalities) 


\section{RESULTS}

\section{Identification and Characterization of Desrt from Mouse}

We have cloned and characterized a novel member of the ARID family of genes, Desrt. The Desrt ORF (GenBank accession no. AFI 69968) encodes a protein of 743 amino acids with a predicted molecular weight of $83 \mathrm{kD}$ (Fig. 1). The domain spanning amino acid positions 295 to 426 has high similarity with the ARID family of DNA-binding proteins (Herrscher et al. 1995; Gregory et al. 1996). The Desrt sequence shares highest amino acid sequence identity (82\%) over a 246 amino acid stretch with the human MRF2 sequence (modulator recognition factor 2, Genbank accession no. M73837). Furthermore, structural predictions of the Desrt ARID domain suggest it closely resembles the NMR-solved structure for MRF2-ARID, (Yuan et al. 1998), which is composed of six $\alpha$-helices, four loops and no $\beta$-sheets.

Higher levels of sequence similarity are detected between mouse Desrt and human MRF1 and MRF2 relative to other members of the ARID family (Fig. 2A). Because ARID-

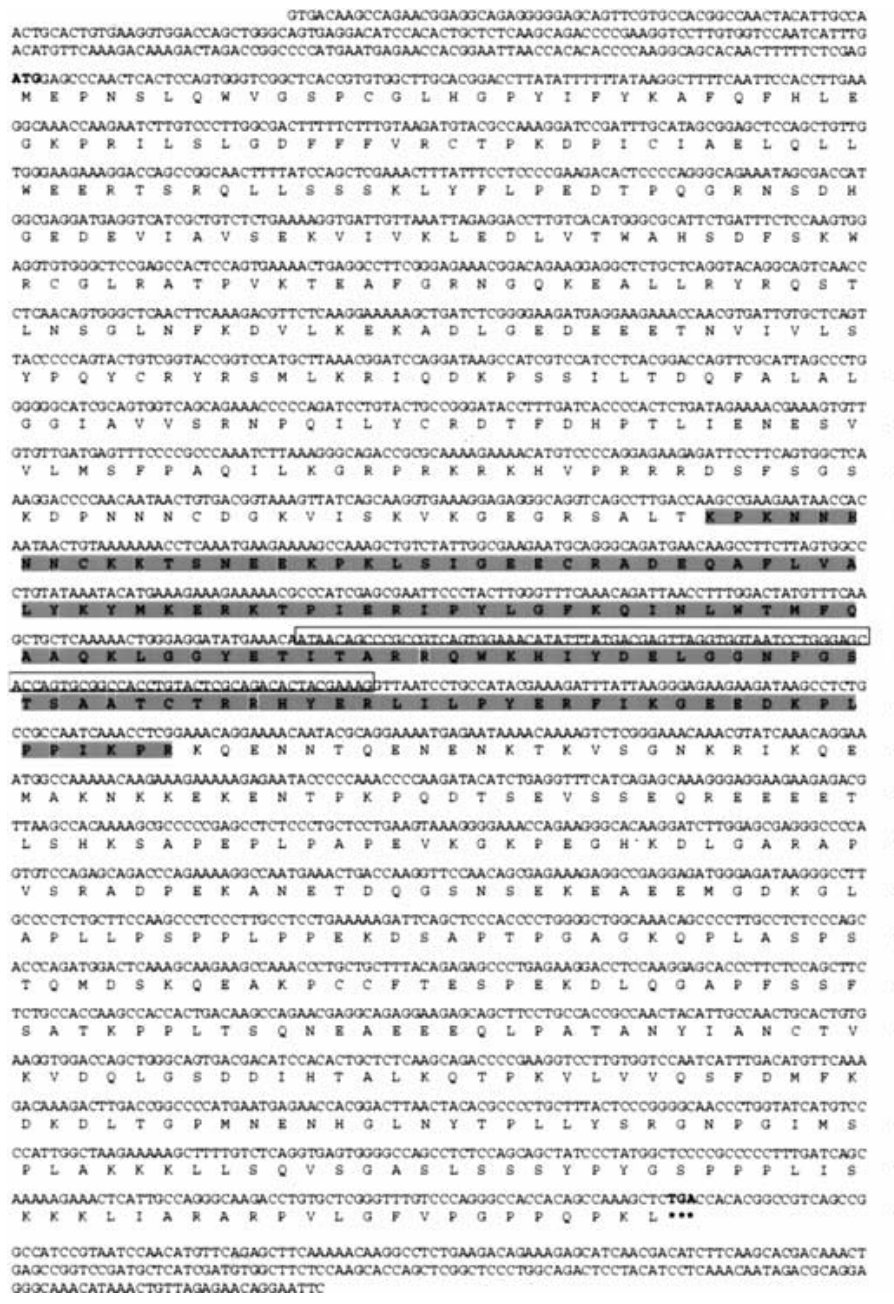

Figure 1 Nucleotide and amino acid sequence of mouse Desrt. Amino acids are numbered and the stop codon is represented by ${ }^{* *}$. The conserved ARID domain (amino acids 295-426) is shaded grey and the 98-bp exon deleted by gene targeting is boxed. containing proteins, including MRF2, Bright, and dri, have been shown to bind AT-rich DNA sequences through their ARID domains (Herrscher et al. 1995; Gregory et al. 1996; Whitson et al. 1999), we tested the ability of Desrt to bind to an AT-rich sequence. A Desrt recombinant polypeptide spanning amino acids 255-451 including the ARID domain binds a $(\mathrm{TAA})_{9}$ repeat oligonucleotide that can be competed in a dose-dependent manner with excess unlabeled oligonucleotide (Complex C1) (Fig. 2B). A lower mobility complex (C2) of weaker intensity was observed that was also competed with excess unlabeled oligonucleotide, and most likely represents a dimeric form of the Desrt recombinant protein. These data show that the Desrt-ARID domain is able to interact with DNA. Furthermore, the Desrt-ARID domain appears to show a preference for binding to AT-rich sequence, as binding to $(\mathrm{TAA})_{9}$ was competed with excess unlabeled (TAA) ${ }_{9}$, but was not competed with excess GC-containing nonspecific oligonucleotide (Fig. 2C).

The expression of Desrt was examined in a number of adult mouse organs by Northern blot. An mRNA transcript of $\sim 9 \mathrm{~kb}$ was detected in poly $(\mathrm{A})^{+}$RNA isolated from a wide variety of adult organs including lung, heart, small intestine, kidney, muscle, and brain (Fig. 3A), suggesting a widespread biological function for Desrt. The expression pattern of Desrt was further characterized by use of
30

60

120 150

180 RT-PCR and Southern blot hybridization by use of an internal oligonucleotide. The increased sensitivity of RT-PCR allowed detection of low levels of Desrt expression undetected by Northern hybridization studies in organs such as the spleen. Thus, expression of Desrt was detected from most organs examined including lung, brain, lymphoid organs such as spleen and thymus, endocrine organs such as the adrenal glands, and some reproductive organs such as uterus and testis. No expression was detected in the ovary by RT-PCR, and although the loading was lower than other organs (as shown by the weaker GAPDH band), even the amplified sensitivity of Southern hybridization failed to detect a signal.

\section{Homozygous Desrt Mutants Have Reduced Viability and are Growth Retarded}

To investigate the biological function of Desrt, we mutated the gene in embryonic stem cells using homologous recombination. A targeting construct was generated by deleting a 98-bp exon within the ARID domain (Fig. 4A). Clones with correctly targeted events were identified and confirmed both by Southern blot hybridization (Fig. 4B) and PCR analysis (Fig. 4C) by use of primers external to the targeting construct (as shown in Fig. 4A), which can only amplify a band of the expected size in clones with targeted events. Desrt expression studies from $+/+,+/-$, and $-/-$ primary embryonic fibroblasts were performed by RT-PCR by use of oligonucleotides in coding regions flanking the targeted exon (Fig. 4D). Southern hybridization of the amplified fragments with internal oligonucleotides (Fig. 4E) and sequencing of the amplified fragments (data not shown) confirmed deletion of the 98-bp exon of the Desrt ARID domain, resulting in a transcriptional frameshift generating stop codons.

Breeding of Desrt heterozygous (+/-) mice indicated that homozygous mutant $(-/-)$ Dest concep- 


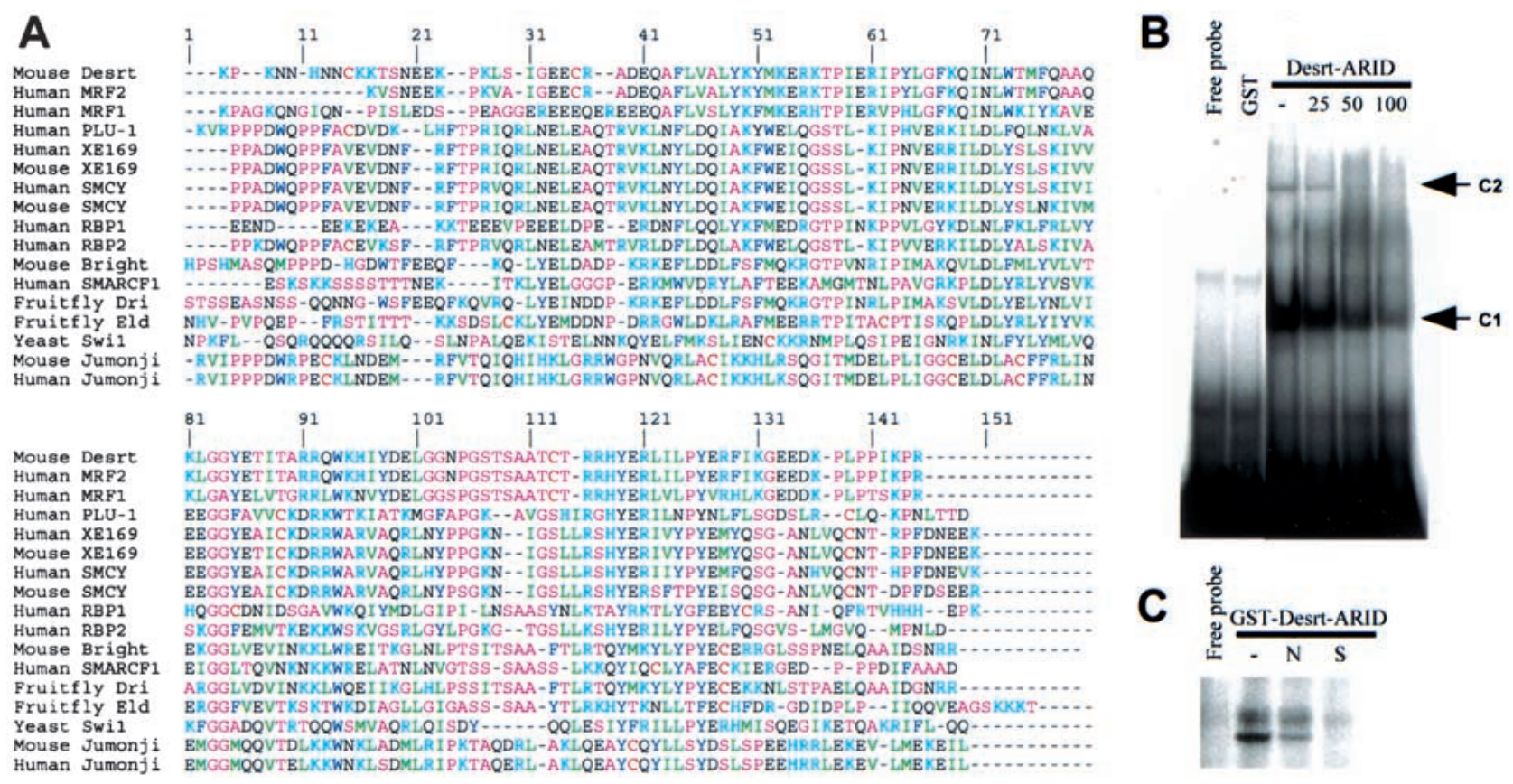

Figure 2 (A) The alignment of amino acids from ARID domains. Aromatic amino acids ( $,, Y, W)$ are dark blue; sulphydrylic amino acids (C) are red; basic amino acids $(K, R, H)$ are light blue; aliphatic amino acids $(V, I, L, M)$ are green; hydrophilic (1) amino acids $(P, A, G, S, T)$ are pink; hydrophilic (2) amino acids (N, E, D, Q) are black. (B) Electrophoretic mobility shift assay (EMSA) of recombinant Desrt-ARID domain binding to a (TTA), oligonucleotide (lane 3). The main Desrt--retarded complex is indicated by C1 and a lower mobility complex indicated by C2. Competition was performed with a 25-, 50-, or 100-fold excess of unlabeled oligonucleotide (lanes 4-6). No binding was observed by use of GST alone (lane 2) or in the absence of protein (lane 1, free probe). (C) EMSA of recombinant GST-Desrt-ARID fusion protein binding to a (TTA), oligonucleotide (lane 2). Competition was performed with a 100-fold excess of unlabeled nonspecific $(\mathrm{N})$ oligonucleotide (lane 3 ) or specific (S) oligonucleotide (lane 4). No binding was observed in the absence of protein (lane 1, free probe).

tuses had reduced viability. At $1 \mathrm{~d}$ postpartum, the ratio of genotypes observed from heterozygous crosses were $+/+=1.0$; $+/-=2.2$, and $-/-=0.4 ; n=71$, indicating a reduced number of homozygous mutants compared with the expected. At weaning (3-wk old), the ratio from heterozygous crosses were $+/+=1.0,+/-=2.1$, and $-/-=0.5(n=395)$, suggesting there was no additional deaths from 1-d postpartum to weaning, and that death of homozygous mutants occured either in utero or within a few hours after birth. Furthermore, aging the mice until 1 yr of age indicated that there was no additional differences in survival between the homozygous mutants that had survived birth and their wild-type counterparts.

The mean weight of Desrt ${ }^{-/-}$mice within the first $24-\mathrm{h}$ postpartum was $80 \%$ that of their wild-type or heterozygous littermates (Fig. 5A), indicating that targeted mice were growth retarded in utero. Desrt ${ }^{-1-}$ mice that survived after birth were observed to be smaller in size than their heterozygous or wild-type control littermates (Fig. 5B) and appeared

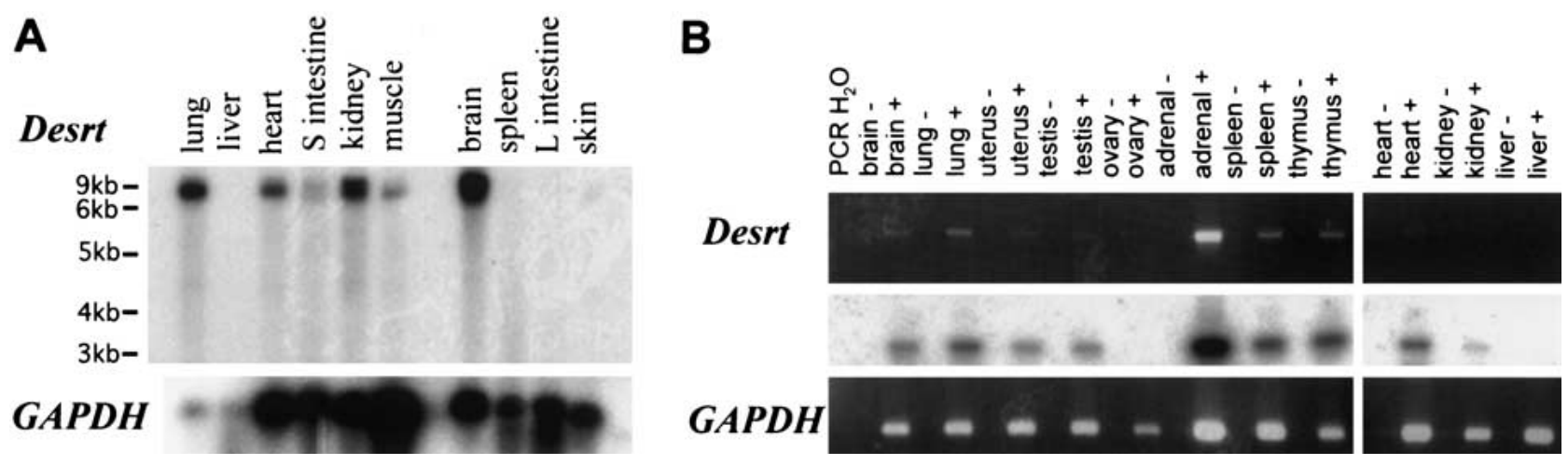

Figure 3 (A) Northern blot analysis of Desrt mRNA expression showing a transcript of $\sim 9 \mathrm{~kb}$ in poly $(A)^{+}$RNA isolated from adult mouse organs. GAPDH was used as a loading control. (B) RT-PCR of Desrt mRNA expression in adult mouse organs. PCR reactions were carried out in the absence of DNA $\left(P C R \mathrm{H}_{2} \mathrm{O}\right)$, or by use of $C D N A$ samples that had been prepared from total RNA in the presence $(+)$ or absence $(-)$ of reverse transcriptase. (Top) RT-PCR amplification of a 619-bp fragment of Desrt on an ethidium bromide-stained agarose gel. Southern blot hybridization of the Desrt PCR fragments by use of an internal oligonucleotide (mDesrt7) was performed (middle). (Bottom) RT-PCR amplification of a 284-bp fragment of GAPDH as a positive control. 
A

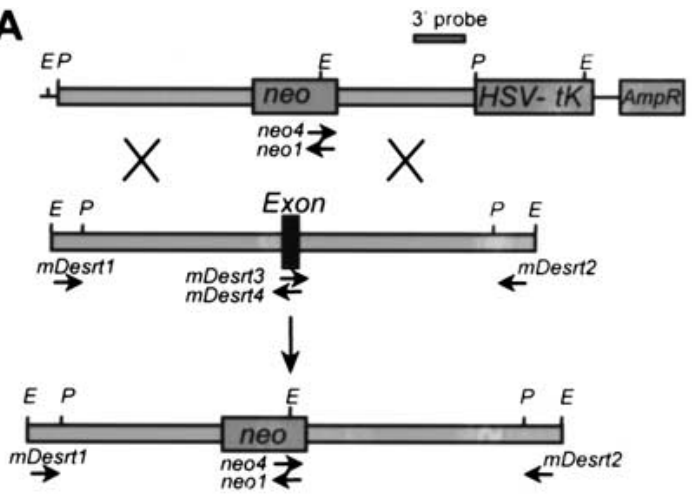

wild-type

allele

targeted

allele

B

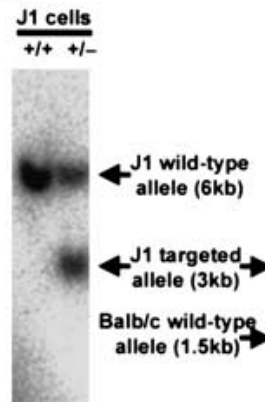

C

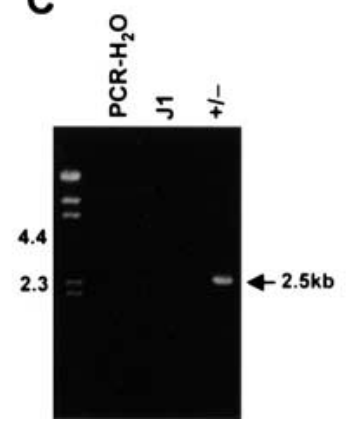

D

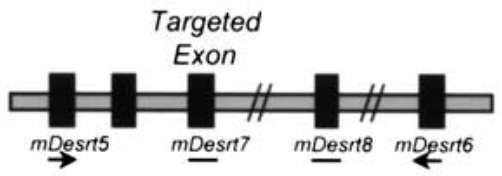

E

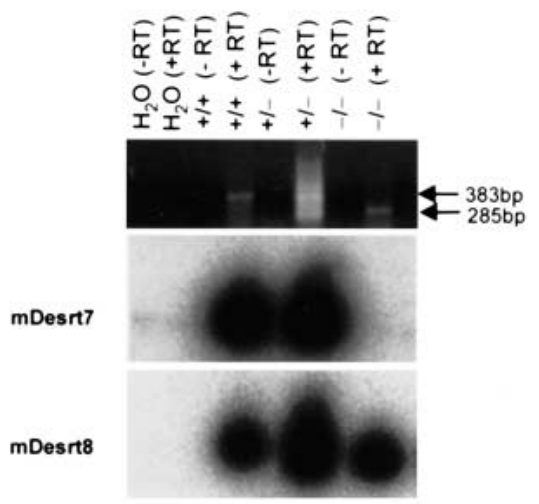

Figure 4 Targeted disruption of the mouse Desrt gene. (A) A schematic representation of a Desrt gene targeting event, showing the positions of ECORI (E) and Pst (P) sites in the vector, the wild-type allele, and the gene-targeted allele. The position of the 3' probe and the oligonucleotides used to confirm the targeting of the allele are indicated. (B) A Southern blot analysis of EcoRI-digested genomic DNA isolated from electroporated ES cell clones (left) and mouse genomic DNA (right) hybridized with the 3' probe. This probe hybridized to a 6-kb EcoRI fragment in the wild-type alleles of unelectroporated J1 ES cells $(+/+)$ and a 3-kb-targeted allele in the heterozygous ES cells $(+/-)$. Progeny of heterozygous matings $(+/-\times+/-)$ are shown at right. The $3^{\prime}$ probe hybridized to a 1.5-kb fragment in EcoRI-digested genomic DNA isolated from wild-type (+/+) $\mathrm{Balb} / \mathrm{c}$ and to a 3-kb targeted allele. (C) PCR analysis of genomic DNA isolated from electroporated ES cell clones by use of oligonucleotide mDesrt2 and a neomycin oligonucleotide, neo4, in the absence of DNA ( $\left.P C \mathrm{H}_{2} \mathrm{O}\right)$, in the presence of unelectroporated J1 DNA, or in the presence of DNA isolated from a Desrt-targeted clone $(+/-)$. A $2.5-\mathrm{kb}$ band was detected in the correctly targeted clone. $(D)$ A schematic representation of a Desrt gene fragment showing the positions of the oligonucleotides designed relative to the targeted exon. Exons are depicted as black boxes. (E) RT-PCR of Desrt mRNA expression in $+/+,+/-$, or $-/-$ primary embryonic fibroblasts. PCR reactions were carried out by use of cDNA samples that had been prepared from total RNA or $\mathrm{H}_{2} \mathrm{O}$, in the presence (+ RT) or absence (- RT) of reverse transcriptase. (Top) A 383-bp +/+ fragment, a 285-bp - / - fragment, or both fragments in +/ - fibroblasts. Southern blot hybridization of the Desrt PCR reactions was performed by use of mDesrt7, an internal oligonucleotide derived from the targeted exon (middle) or mDesrt8, from a nontargeted exon (bottom). This showed that the PCR-amplified fragments derived from RNA of wild-type or targeted alleles hybridized to internal oligonucleotides derived from the nontargeted exon, whereas the PCR fragments derived from the targeted allele did not hybridize to the internal oligonucleotide derived from the targeted exon.

emaciated with a sparse, ungroomed coat. Desrt ${ }^{-/-}$mice had a significantly reduced growth rate (measured over days 8-21) at 0.21 grams/day when compared with 0.38 and 0.40 grams/ day in wild-type and heterozygous littermates, respectively (Fig. 5C). Thus, the growth retardation observed at birth became more pronounced as postnatal growth proceeded, such that by $6.5 \mathrm{wk}$, the mean weight of the Desrt ${ }^{-/-}$mice was $69 \%$ that of the wild-type littermates (Fig. 5D). The postnatal growth rates, growth retardation, and survival rates were consistent in male and female Desrt ${ }^{-/-}$mice (data not shown). The Desrt ${ }^{-1-}$ mice do not recover to the size and weight of the wild-type and heterozygous animals with further aging (data not shown).

Desrt $^{- \text {- }}$ Mice Display Abnormalities of the Male and Female Reproductive Organs and the Adrenal Glands Desrt $^{-/-}$mice exhibit reduced mating behavior with severely reduced rates of vaginal plug formation after caging Desrt $^{-/-}$ males with wild-type females (data not shown). Furthermore, Desrt $^{-/-}$males were found to be either unilaterally or bilat- erally cryptorchid, with undescended testes generally located in the inguinal region (Fig. 6A) The undescended testes of Desrt $^{-/-}$mice were smaller than descended testes of Desrt ${ }^{-/-}$ and wild-type littermates (Fig. 6B). Histological examination of the cryptorchid testes from Desrt ${ }^{-1-}$ mice indicated that there was a high incidence of markedly disrupted spermatogenesis as compared with wild-type littermates (Fig. 6C,D). There was a decrease in numbers of all germ-cell types, particularly affecting the post-meiotic spermatid population. Round and elongated spermatids were rarely observed, and many cells at the luminal border of the epithelium were degenerating, often with the formation of multinucleated giant cells (symplasts) as compared with controls. Spermatogenesis in the descended testis of Desrt ${ }^{-/-}$mice appeared normal.

Desrt $^{-1-}$ females also have reduced rates of vaginal plug formation when caged with wild-type males (data not shown). Histological examination from 4-m-old heterozygote and Desrt ${ }^{-/-}$females revealed the absence of corpora lutea in the latter with follicular development to the antral stage only as compared with the heterozygote mice in which normal 

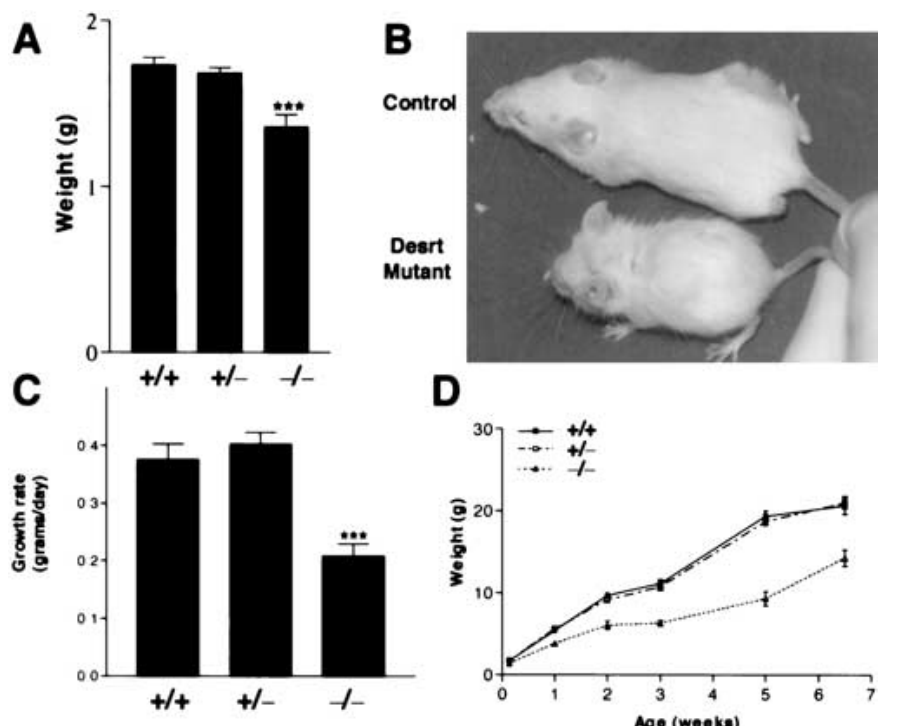

Figure 5 Growth retardation of Desrt ${ }^{-/-}$mice. (A) Weight (mean \pm SEM) of neonatal Desrt ${ }^{+/+}(n=19)$, Desrt ${ }^{+/-}(n=38)$, and Desrt ${ }^{-/-}(n=7)$ mice (*** $p<0.001)$. (B) A 26-d-old Desrt ${ }^{-1-}$ mouse and its sex-matched control littermate showing the reduced size, the sparse, ungroomed coat, and the emaciated appearance of the Desrt ${ }^{-/-}$. (C) Growth rate of Desrt $\mathrm{H}^{+/+}(n=16)$, Desrt ${ }^{+/-}$ $(n=24)$, and Desrt ${ }^{-/-}(n=8)$ mice, measured over 8-21 d (*** $\left.p<0.001\right) .(D)$ Weight of Desrt ${ }^{+/+}$, Desrt ${ }^{+/-}$, and Desrt ${ }^{-/-}$mice from day 1 to week $6.5(n=7-$ 38).

follicular development occurred (Fig. 6E,F). Histological examination of the uterine horns of Desrt ${ }^{-/-}$females revealed a reduction in the overall size of the uterine horns, which reflected a reduction in the thickness of the muscularis layers and the uterine stroma or strata basalae as compared with the heterozygote uterus (Fig. 6G,H). In addition, the Desrt ${ }^{-/-}$ uterine glands appeared smaller in size (Fig. 6G,H).

Histopathological analysis of other organs of Dest ${ }^{-1-}$ mice revealed abnormalities in the adrenal gland. Whereas the morphology of cell types in the adrenal appeared normal, a significant reduction in the thickness of the zona reticularis was consistently detected as compared with age and sexmatched controls (Fig. 6I,J). The medulla, zona fasiculata, and the zona glomerulosa were not consistently altered between knockout mice and their wild-type counterparts.

\section{Desrt $^{-/-}$Mice Develop Transient}

\section{Immune Abnormalities}

Histological examination of the haematopoietic organs of 4-wk-old Desrt ${ }^{-1-}$ mice revealed a smaller thymus and spleen than in the wild-type littermates. Thymic architecture was disrupted with thinning of the cortex associated with a reduction in the lymphocytes within the cortex (Fig. 7A,B), although spleen architecture was normal (data not shown).

In 3-wk-old Desrt ${ }^{-1-}$ mice, significant reductions in the femoral bone marrow (BM), spleen, and thymic cellularities were observed, compared with their wild-type littermates (Fig. 7C). In the thymus, there was a significant decrease in the percentage of $\mathrm{CD}^{+} \mathrm{CD}^{+}$double-positive thymocytes $(77.5 \%$ in Desrt $^{+++}, 15.9 \%$ in Desrt ${ }^{-/-}$) with a corresponding increase in the percentage of $\mathrm{CD}^{+} \mathrm{CD}^{-}\left(13.8 \%\right.$ in Desrt $^{+/+}, 49.7 \%$ in Desrt $\left.^{-/-}\right)$and $\mathrm{CD}^{-} \mathrm{CD}^{+}$(6.2\% in $\mathrm{Desrt}^{+/+}, 31.2 \%$ in Desrt $^{-/-}$) mature single-positive thymocytes. B cell differentiation within the BM was similarly affected in Desrt ${ }^{-/-}$mice with significantly reduced proportions of $\mathrm{B}^{2} 2 \mathrm{O}^{+} \mathrm{IgM}^{-}$ cells $\left(38.7 \%\right.$ in Desrt $^{+/+}, 9.0 \%$ in Desrt $\left.{ }^{-/-}\right)$, which encompasses the early $B$ cell progenitors to preB cells. In the spleen, there was no change in the proportions of lymphoid and myeloid cells (data not shown).

In 6-wk-old mice, both the cellularities and $\mathrm{T}$ and B cell proportions of spleen and thymus of Desrt ${ }^{-/-}$ mice were not significantly different from that of wildtype controls, whereas the BM cellularities of Desrt ${ }^{-/-}$ mice remained below the levels of wild-type littermates. However, despite the reduced BM cellularity, the $\mathrm{BM}$ appeared normal in that the incidence of progenitors in the BM was identical to controls (data not shown). These data suggest that young Desrt ${ }^{-/-}$mice display transient abnormalities in their lymphoid organs that recover with age.

\section{DISCUSSION}

We have cloned and characterized mouse Desrt, a novel gene encoding a member of the newly identified ARID class of DNA-binding proteins, which is required for growth and the development of reproductive organs and the immune system in the mouse. The preference for binding to AT-rich sequences and the high levels of sequence similarity observed between the ARID domains of family members suggests that Desrt is likely to interact with DNA via minor and major groove interactions, similar to Bright and MRF2 (Herrscher et al. 1995; Whitson et al. 1999).

The binding of AT-rich sequences in the minor groove may represent a common feature of transcription factors that play a dual role in regulating specific gene expression and in chromatin reorganization. The HMGI proteins, which bind DNA in a similar manner (Reeves et al. 1990), have been proposed to modulate chromatin structure by inducing DNAbinding and enabling the binding of other transcription factors (Falvo et al. 1995; Mantovani et al. 1998). Furthermore, the mouse mutant for HMGI-C (pygmy) displays both cryptorchidism and prenatal and postnatal growth retardation similar to the mutant Desrt mouse (Benson et al. 1994; King et al. 1955; Zhou et al. 1995). The similarity in DNA-binding properties between ARID members and HMGI-C and the similarity in phenotypes between the Desrt ${ }^{-/-}$and the pygmy mice suggests that the phenotypes observed in Desrt mutants may be related to abnormalities in chromatin remodeling.

Reduced viability was also observed in the Desrt ${ }^{-/-}$mice, suggesting that the Desrt mutation is embryonic or perinatal lethal for $50 \%$ of the targeted mice. This survival rate may relate to the fact that the mice investigated in this study were of a mixed genetic background $(129 / \mathrm{Sv} \times \mathrm{Balb} / \mathrm{c})$ and will be further analyzed after backcrossing the Dest mutants onto pure genetic backgrounds. Importantly, we also note that mutations in other ARID members examined have been embryonic lethal both in Drosophila eld/osa, dri, and in mouse Jumonji mutants (Takeuchi et al. 1995; Treisman et al. 1997; Shandala et al. 1999; Vazquez et al. 1999), indicating the importance of Desrt and other ARID proteins in development.

Other abnormalities observed in the Desrt ${ }^{-/-}$mice include reduced fertility, adrenal abnormalities, and transient immune abnormalities. Immune abnormalities have been associated with dysregulation of adrenal hormones (Boehme et al.1997), whereas the reduced fertility is consistent with reproductive abnormalities observed histologically. The cessa- 

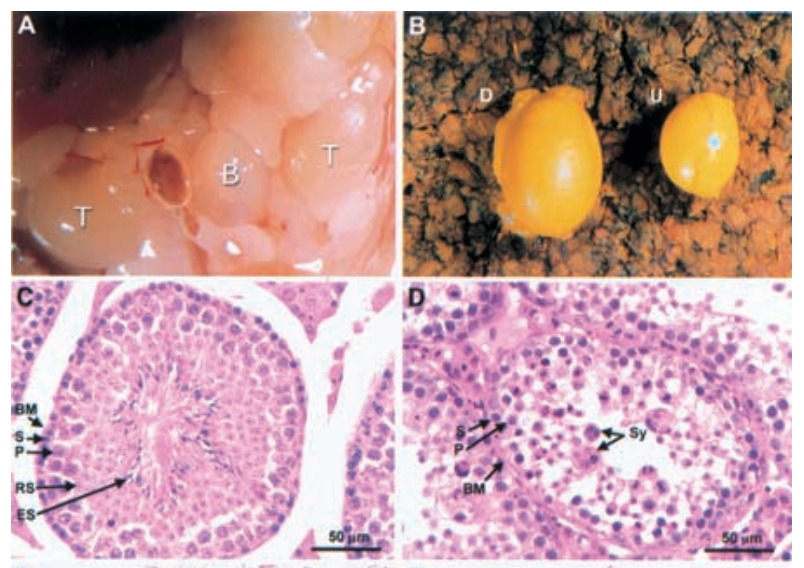

E
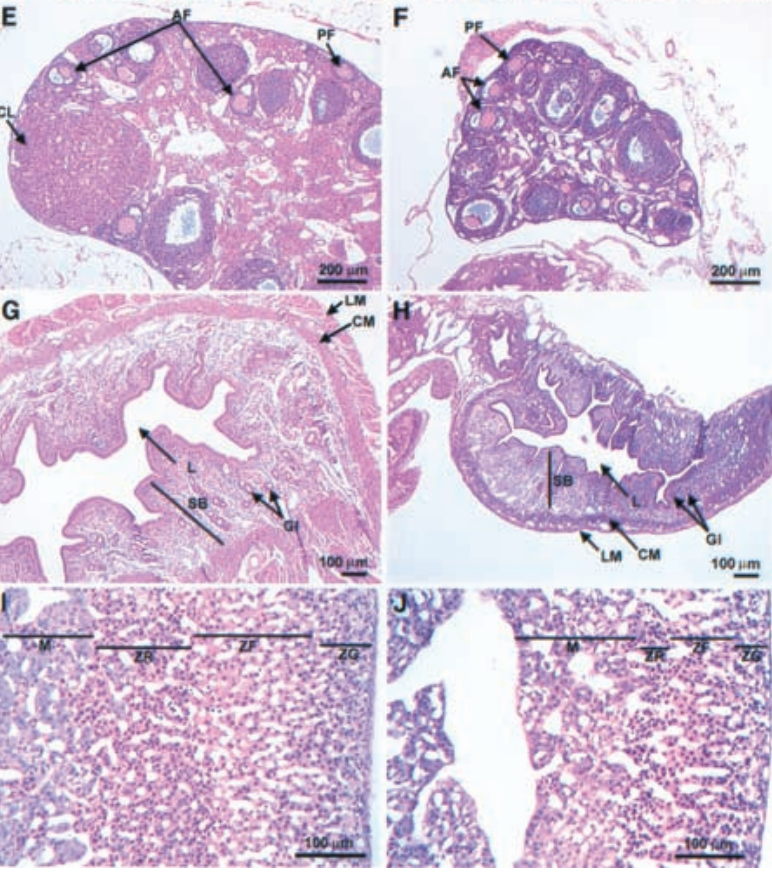

Figure 6 Pathology of the Desrt ${ }^{-1-}$ mice. (A) Bilaterally cryptorchid testes of a 6.5 -wk-old Desrt ${ }^{-1-}$ mouse. The undescended testes (T) are shown relative to the position of the bladder (B). (B) Descended $(D)$ and undescended $(U)$ testis, of a 6.5-wk-old unilaterally cryptorchid Desrt ${ }^{-/-}$mouse showing the smaller size of the undescended testis. $(C, D)$ Histopathology of a 14-wk-old testis. Seminiferous tubules of a wild-type testis (C) densely packed with spermatogenic cells as compared with an undescended Desrt ${ }^{-1-}$ testis $(D)$ with disturbed spermatogenesis shown by the marked decrease in the number of germ cells, particularly the round spermatids (RS) and elongated spermatids (ES) and the presence of symplasts (Sy). (S) Spermatogonia; $(P)$ primary spermatocytes; $(B M)$ basement membrane. $(E, F)$ Histopathology of a 4-mo-old ovary from a heterozygous mouse $(E)$ showing normal follicular maturation as compared with a Desrt ${ }^{-\prime-}$ ovary $(F)$ showing the absence of corpora lutea. (PF) Primary follicles; (AF) secondary antral follicles; $(\mathrm{CL})$ corpora lutea. $(G, H)$ Histopathology of a 4-mo-old uterus from a heterozygous mouse $(G)$ and the markedly smaller Desrt ${ }^{-1-}$ uterus $(H)$ showing thinner muscle layers, a reduced stratum basalae (SB), containing smaller glands, (G1). (LM) Longitudinal muscle; (CM) circular muscle; (L) uterine lumen. $(I, D)$ Histopathology of 4-wk-old female (I) control and (J) Desrt ${ }^{-/-}$adrenal glands showing the severely diminished size of the zona reticularis. (M) Medulla; (ZR) zona reticularis; (ZF) zona fasiculata; (ZG) zona glomerulosa.
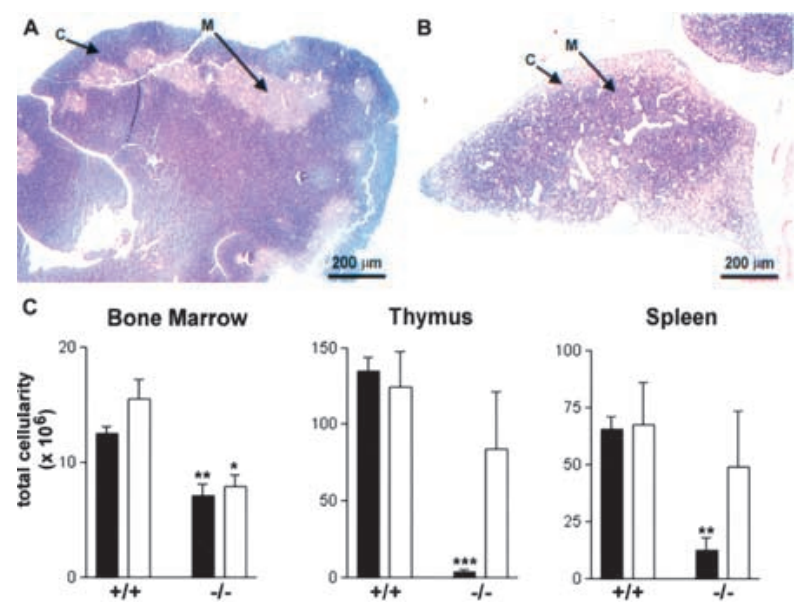

Figure $7 \quad(A, B)$ Histopathology of 4-wk-old female $(A)$ control and (B) Desrt ${ }^{-/-}$thymi showing the overall reduction in the size of the Desrt $^{-1-}$ thymus, the paler staining of the thymic cortex (C) and the darker stain of the thymic medulla (M) as compared with the control. (C) Comparison of the femoral bone marrow, thymus, and spleen cellularities of Desrt ${ }^{+/+}$and Desrt ${ }^{-/}$mice at 3-wk of age (solid bars) and at 6 wk (open bars). The cellularities of Desrt ${ }^{-1-}$ animals were compared with the cellularity of age matched Desrt ${ }^{+/+}$animals and significant differences are indicated $\left({ }^{*}, p<0.05 ;{ }^{* *}, p<0.01 ;{ }^{* * *}\right.$, $p<0.001)$.

tion of ovarian follicular maturation at the antral stage indicates the failure of ovulation, supported by the absence of corpora lutea. These results would be consistent with the atrophic appearance of the endometrium, presumably due to the diminished estrogenic and absent progestagenic stimulation. The failure of ovulation may be related to the lower body mass associated with growth retardation or may be mediated by failure of cyclic gonadotrophic stimulation.

Male Desrt ${ }^{-/-}$mice also showed reduced mating behavior and abnormalities in testicular descent. Normal testicular descent is proposed to occur in two stages involving (a) migration from the abdomen, which is mediated by Mullerian Inhibitory Substance and swelling of the gubernaculum, and (b) the androgen dependent migration of the gubernaculum into the scrotum and atrophy of the cranial sensory ligament (for review, see Hutson et al. 1997). The undescended testis of Desrt $^{-/-}$mice was often found in the inguinal region, suggesting that the first stage of testicular descent occurred normally, but the secondary stage was incomplete. Hypospermatogenesis was observed in some undescended testes and is presumed to be a consequence of cryptorchidism, as failure of testicular descent into the scrotum leads to secondary degeneration and reduced fertility. The secondary nature of the spermatogenic abnormalities was supported by evidence that in cases of unilateral cryptorchidism in Desrt ${ }^{-/-}$mice, the descended testis appeared normal in size and histology.

A similar phenotype of growth retardation, reduced viability, and abnormalities of the female reproductive organs has been observed in mice with mutations for genes known to function in the growth hormone (GH)-insulin-like growth factor (IGF) pathway. Similar immune abnormalities have been reported for the mouse dwarf mutant Snell, a mutant for pit1, which acts upstream of GH in the GH-IGF axis (Cross et al. 1992). Furthermore, mutants for IGF1, which functions as a mediator of $\mathrm{GH}$ action postnatally but is $\mathrm{GH}$ independent during embryogenesis, are severely growth retarded at birth

\section{Genome Research}


and continue to grow with a retarded growth rate (Baker et al. 1993; Powell-Braxton et al. 1993). They also display reduced viability with neonatal deaths occuring at a frequency of 32\%-95\% dependent on genetic background (Liu et al. 1993; Powell-Braxton et al. 1993) and show strikingly similar uterine and ovarian abnormalities (Baker et al. 1996). Although abnormalities in testicular descent have not been described for the IGF1 mutants, recent studies indicate that targeted disruption of the gene encoding Leydig cell insulin-like hormone (designated Insl3) causes cryptorchidism with the testes retained close to the kidney (Nef and Parada 1999; Zimmermann et al. 1999). Furthermore, the Insl3 heterozygous males displayed delayed testicular descent, although this was corrected in the adult (Nef and Parada 1999), suggesting a gene dosage-dependent effect of Insl3. Thus, although it is unlikely that Desrt acts directly on Insl3, the phenotype observed in the Desrt mutants may be consistent with a role for Desrt in the GH-IGF pathway, and Desrt may interact with other insulin-like molecules to effect testicular descent.

In conclusion, we have cloned and characterized a novel gene, Desrt, a member of the recently identified ARID family of DNA-binding proteins. We have established the importance of this gene in growth, immune, and sexual development. The similarity in phenotypes between the Desrt ${ }^{-1-}$ mouse and the described mouse mutants, suggests that Desrt may be effecting its biological function through several different pathways that may include the GH/IGF pathway or other insulin-like molecules. Further studies will focus on the identification of these pathways to determine the role of Desrt in transcriptional regulation of development

\section{METHODS}

\section{Cloning and Characterization of Murine Desrt cDNA}

A partial cDNA fragment (375 bp) of murine Desrt, designated clone BL21 (Corrick et al. 1996), was used to identify overlapping fragments in a XZAPII murine lung cDNA library (Stratagene) and the full-length ORF was subsequently identified by $5^{\prime}$ and 3' RACE (Marathon RACE Kit, Stratagene).

The mouse Desrt ARID domain was aligned to those in Human (MRF2-M73837; MRF1-M62324; RBP1-S66427; RBP2S66431; Jumonji-U57592; XE169-L25270; SMCY-U52191), murine (Jumonji-D31967; XE169-Z29651; SMCY-Z29652; Bright-U60335), fruitfly (dri-U62542; eld-2981221), and yeast ( Swi1-U33335) (accession numbers follow the hyphens) by use of CLUSTAL W (Thompson et al. 1994) with default settings invoked, then improved manually by use of the $\mathrm{Ge}-$ netic Database Environment program (Smith et al. 1994).

Desrt DNA-binding activity to double-stranded (TAA) oligonucleotide was measured by use of either $1.5 \mu \mathrm{g}$ of thrombin-cleaved Desrt-GST fusion protein (amino acids 255-451 of Desrt), Desrt-GST fusion protein, or GST protein alone and 20 pmole of $\left[\gamma^{32} \mathrm{P}\right]$ ATP oligonucleotide, followed by $6 \%$ PAGE. Competition was carried out with 25-, 50-, or 100fold excess of unlabeled-specific (TAA) ${ }_{9}$ oligonucleotide or nonspecific oligonucleotide (ATCTTCTCCGGGTGCTT).

Poly $(\mathrm{A})^{+}$mRNA from murine organs was prepared and analyzed by Northern blot hybridization (Owczarek et al. 1997) with ${ }^{32}$ P-labeled cDNA of murine Dest and rat GAPDH.

Reverse-transcription reactions were carried out by use of random primers and $2 \mu \mathrm{g}$ of total RNA (Omniscript, Qiagen) according to the manufacturer's instructions. PCR reactions were then carried out to amplify a 284-bp fragment for GAPDH (forward primer, GAACGGGAAGCTTGTCAT CAATGG, reverse primer, CTAAGCAGTTGGTGGTGCAG) and a 619-bp fragment for Desrt (mDesrt9 forward primer,
GGAAGAGATTCCTTCAGT, mDesrt6 reverse primer, CGCTCGAGGGGTTTGGGGGTATTCTC). Southern hybridization of the Desrt PCR reactions was performed with an internal oligonucleotide (mDesrt7, GTTAGGTGGTAATCCTGGGAGC).

\section{Generation of a Targeting Vector and Desrt ${ }^{-/-}$Mice}

A 16-kb genomic fragment was identified from a $\lambda$ fixII murine $129 /$ SvJ genomic DNA library (Stratagene) that contained two exons spanning the ARID sequence, separated by a 9-kb intron. A 5-kb PstI genomic fragment containing a 98-bp exon (nucleotide positions 1350-1447, contained within ARID) was subcloned, and the replacement vector generated by excision of a 400-bp SacII/BstEII fragment containing the exon and insertion of a XhoI/SalI neo cassette (derived from pMC1neoPolyA, Stratagene) in the reverse orientation. A HSV-tk (XhoI/HindIII fragment from pMC1-TK) cassette was cloned in the reverse orientation into the HindIII site of the vector polylinker, the resultant targeting construct linearized and electroporated into $\mathrm{J} 1$ embryonic stem cells ( $\mathrm{Li}$ et al. 1992), which were selected by use of $300 \mu \mathrm{g} / \mathrm{mL}$ G418 and 2 $\mu \mathrm{M}$ ganciclovir. Clones were screened for a targeting event by long-range PCR using Elongase (GIBCO BRL). Intronic primers were designed external to the targeting vector and used with either an internal (exon-specific) oligonucleotide or a neomycin oligonucleotide in a separate reaction as shown in Figure 4A. The 3' intronic primer mDesrt2 (GTTGC TAGGGCTTTCCAAATG) amplified a $2.7-\mathrm{kb}$ product with mDesrt3 (GTTAGGTGGTAATCCTGGGAGC) or a 2.5 -kb product with neo4 (CATTGGGTGGAAACATTCCAGG). Targeted events were verified by Southern blot analysis of EcoRIdigested DNA. Targeted cells were microinjected into blastocycts derived from Balb/c mice and transferred into pseudopregnant Balb/c mice. Desrt ${ }^{+/-}$mice were generated by cross breeding germ-line chimaeric mice with Balb/c, confirmed by Southern blot analysis, and used to generate Desrt ${ }^{-1-}$ mice. RT-PCR reactions were carried out to amplify a 383-bp wildtype fragment or a 285-bp targeted fragment for Desrt (mDesrt5 forward primer CCATCGAGCGAATTCCCTAC, mDesrt6 reverse primer). Southern hybridization of the PCR reactions was performed by use of an internal oligonucleotide from the targeted exon (mDesrt7) or from a nontargeted exon (mDesrt8, GGCCATTTCCTGTTTGATACG). All animals were maintained in a conventional animal house. Mouse testis were defined as cryptorchid only if the testis could not be physically descended from the inguinal into the scrotal region. Histopathology was performed on haematoxylin and eosin-stained sections (4 micron) cut from mouse organs fixed in $10 \%$ phosphate-buffered formalin. Immunophenotyping was performed by use of single cell suspensions prepared from the spleen, thymus, femoral bone marrow, and peripheral blood of knockout mice and their littermate controls. Cellularities were determined (Sysmex K1000 haemalyser) and flow cytometric analysis performed (FACStarplus, Becton Dickinson) by use of a panel of monoclonal antibodies to quantify B cell (anti-B220, clone RA3.6B2; anti-IgM; anti-IgD) and T cell lineages (anti- CD4, clone GK1.5; CD8, clone 53-6.7).

\section{ACKNOWLEDGMENTS}

We thank Ernst Wolvetang, Trevor Wilson, Francesca Lazner, Rocco Iannello, Cathie Corrick, Dennis Engler, Christine Rodda, and Anne O'Connor for helpful discussions and comments, and Pamela Farmer for assistance with histological examination of testicular descent. This project was supported by an Australian Research Council grant.

The publication costs of this article were defrayed in part by payment of page charges. This article must therefore be hereby marked "advertisement" in accordance with 18 USC section 1734 solely to indicate this fact. 


\section{REFERENCES}

Baker, J, Liu, J-P, Robertson, E.J., and Efstratiadis, A.1993. Role of insulin-like growth factors in embryonic and postnatal growth. Cell 75: 73-82.

Baker, J., Hardy, M.P., Zhou, J., Bondy, C., Lupu, F., Bellve, A.R., and Efstratiadis, A. 1996. Effects of an Igf1gene null mutation on mouse reproduction. Mol. Endocrinol. 10: 903-918.

Benson, K.F. and Chada, K. 1994. Mini-mouse: Phenotypic characterization of a transgenic mutant allelic to pygmy. Genet. Res. Camb. 64: 27-33.

Boehme, S.A., Gaur, A., Crowe, P.D., Liu, X.J., Tamraz, S., Wong, T., Pahuja, A., Ling, N., Vale, W., De Souza, E.B., et al. 1997. Immunosuppressive phenotype of corticotropin-releasing factor transgenic mice is reversed by adrenalectomy. Cell. Immunol. 176: $103-112$.

Chiba, H., Muramatsu, M., Nomoto, A., and Kato, H. 1994. Two human homologues of Saccharomyces cerevisiae SWI2/SNF2 and Drosophila brahma are transcriptional coactivators cooperating with the estrogen receptor and the retinoic acid receptor. Nucleic Acids Res. 22: 1815-1820.

Corrick, C.M., Silvestro, M.J., Lahoud, M.H., Allen, G.J., Tymms, M.J., and Kola, I. 1996. Construction of a mouse blastocyst cDNA library by PCR amplification from total RNA. Mol. Reprod. Dev. 43: 7-16.

Cross, R.J., Bryson, J.S., and Roszman, T.L. 1992. Immunologic disparity in the hypopituitary dwarf mouse. J. Immunol. 148: $1347-1352$.

Dallas, P.B., Cheney, I.W., Liao, D-W., Bowrin, V., Byam, W. Pacchione, S., Kobayashi, R., Yaciuk, P., and Moran, E. 1998. p300/CREB binding protein-related protein p270 is a component of mammalian SWI/SNF complexes. Mol. Cell. Biol. 18: 3596-3603.

Falvo, J.V., Thanos, D., and Maniatis, T. 1995. Reversal of intrinsic DNA bends in the IFN $\beta$ gene enhancer by transcription factors and the architectural protein HMG I(Y). Cell 83: 1101-1111.

Gregory, S.L., Kortschak, D., Kalionis, B., and Saint, R. 1996. Characterization of the dead ringer gene identifies a novel, highly conserved family of sequence-specific DNA binding proteins. Mol. Cell. Biol. 16: 792-799.

Herrscher, R.F., Kaplan, M.H., Lelsz, D.L., Das, C., Scheuermann, R., and Tucker, P.W. 1995. The immunoglobulin heavy-chain matrix associating regions are bound by Bright: A B cell-specific transactivator that describes a new DNA binding protein family. Genes \& Dev. 9: 3067-3082.

Hutson, J.M., Hasthorpe, S., and Heyns, C.F. 1997. Anatomical and fuctional aspects of testicular descent and cryptorchidism. Endocr. Rev. 18: 259-280.

King, J.W.B. 1955. Observations on the mutant 'pygmy' in the house mouse. Genetics 53: 487-497.

Li, E., Bestor, T.H., and Jaenisch, R. 1992. Targeted mutation of the DNA methyltransferase gene results in embryonic lethality. Cell 69: 915-926.

Liu, J-P., Baker, J., Perkins, A.S., Robertson, E.J., and Efstratiadis, A. 1993. Mice carrying null mutations of the genes encoding insulin-like growth factor 1(Igf-1) and type 1 Igf-receptor (Igf1r). Cell 75: 59-72.

Mantovani, F., Covaceuszach, S., Rustighi, A., Sgarra, R., Heath, C., Goodwin, G.H., and Manfioletti, G. 1998. NF-кB mediated transcriptional activation is enhanced by the architectural factor HMGI-C. Nucleic Acids Res. 26: 143-1439.

Nef, S. and Parada, L.F. 1999. Cryptorchidism in mice mutant for Insl3. Nat. Genet. 22: 295-299.
Owczarek, C.M., Hwang, S.Y., Holland, K.A., Gulluyan, L.M., Tavaria, M., Weaver, B., Reich, N.C., Kola, I., and Hertzog, P.J. 1997. Cloning and characterization of the soluble and transmembrane isoforms of a novel component of the murine type I interferon receptor, IFNAR2. J. Biol. Chem.

272: 23865-23870.

Peterson, C.L., Dingwall, A., and Scott, M.P. 1994. Five SWI/SNF gene products are components of a large multisubunit complex required for transcriptional enhancement. Proc. Natl. Acad. Sci. 91: $2905-2908$.

Powell-Braxton, L., Hollingshead, P., Warburton, C., Dowd, M., Pitts-Meek, S., Dalton, D., Gillett, N., and Stewart, T.A. 1993. IGF-I is required for normal embryonic growth in mice. Genes \& Dev. 7: 2609-2617.

Reeves, R. and Nissen, M.S. 1990. The A T-DNA-binding domain of mammalian high mobility group I chromosomal proteins. J. Biol. Chem. 265: 8573-8582.

Shandala, T., Kortschak, R.D, Gregory, S., and Saint, R. 1999. The Drosophila dead ringer gene is required for early embryonic patterning through regulation of argos and buttonhead expression. Development 126: 4341-4349.

Smith, S.W., Overbeek, R., Woese, C.R., Gilbert, W., and Gillevet, P.M. 1994. The genetic data environment an expandable GUI for multiple sequence analysis. Comput. Appl. Biosci. 10: 671-675.

Takeuchi, T., Yamazaki, Y., Katoh-Fukui, Y., Tsuchiya, R., Kondo, S., Motoyama, J., and Higashinakagawa, T. 1995. Gene trap capture of a novel mouse gene, jumonji, required for neural tube formation. Genes \& Dev. 9: 1211-1222.

Thompson, J.D., Higgins, D.G., and Gibson, T.J. 1994. CLUSTAL W: Improving the sensitivity of progressive multiple sequence alignment through sequence weighting, position-specific gap penalties and weight matrix choice. Nucleic Acids Res. 22: $4673-4680$

Treisman, J.E., Luk, A., Rubin, G.M., and Heberlein, U. 1997. eyelid antagonizes wingless signaling during Drosophila development and has homology to the Bright family of DNA-binding proteins. Genes \& Dev. 11: 1949-1962.

Valentine, S.A., Chen, G., Shandala, T., Fernandez, J., Mische, S., Saint, R., and Courey, A.J. 1998. Dorsal mediated repression requires the formation of a multiprotein repression complex at the ventral silencer. Mol. Cell. Biol. 18: 6584-6594.

Vazquez, M., Moore, L., and Kennison, J.A. 1999. The trithorax group gene osa encodes an ARID-domain protein that genetically interacts with the Brahma chromatin-remodelling factor to regulate transcription. Development 126: 733-742

Whitson, R.H., Huang, T., and Itakura, K. 1999. The Novel Mrf-2 DNA-binding domain recognizes a five-base core sequence through major and minor-groove contacts. Biochem. Biophys. Res. Commun. 258: 326-331.

Yuan, Y-C., Whitson, R.H., Liu, Q., Itakura, K. and Chen, Y. 1998. A novel DNA-binding motif shares structural homology to DNA replication and repair nucleases and polymerases. Nat. Struct. Biol. 5: 959-964.

Zimmermann, S., Steding, G., Emmen, J.M. A., Brinkmann, A.O., Nayernia, K., Holstein, A.F., Engel, W., and Adham, I.M. 1999. Targeted disruption of the Insl3 gene causes bilateral cryptorchidism. Mol. Endocrinol. 13: 681-691.

Zhou, X., Benson, K.F., Ashar, H.R., and Chada, K. 1995. Mutation responsible for the mouse pygmy phenotype in the developmentally regulated factor HMGI-C. Nature 376: 771-774.

Received October 23, 2000; accepted in revised form May 2, 2001.

1334 Genome Research 


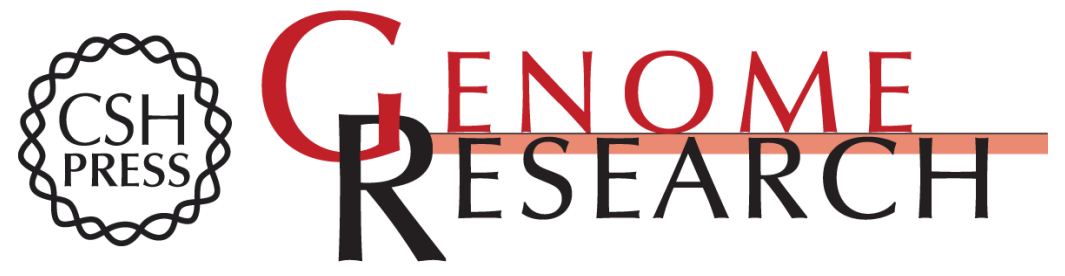

\section{Gene Targeting of Desrt, a Novel ARID Class DNA-Binding Protein, Causes Growth Retardation and Abnormal Development of Reproductive Organs}

Mireille H. Lahoud, Sika Ristevski, Deon J. Venter, et al.

Genome Res. 2001 11: 1327-1334

Access the most recent version at doi:10.1101/gr.168801

\section{License}

Email Alerting

Receive free email alerts when new articles cite this article - sign up in the box at the Service top right corner of the article or click here.

\section{Affordable, Accurate Sequencing.}

